\title{
What is systems biology?
}

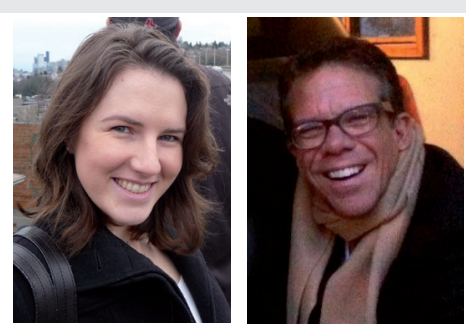

"What truly sets systems biology

apart ... is the use of bioinformatics to

computationally model molecular

networks and define relationships

between pathogen and host."

Jennifer R Tisoncik \& Michael $\mathrm{G}$ Katze $^{\dagger}$

${ }^{\dagger}$ Author for correspondence: University of Washington, Department of Microbiology and Washington National Primate Research Center, Box 358070, Seattle, WA, 98195-8070, USA - Tel.: +1 2067326735

= Fax: +1 2067326056 m money@u.washingon.edu

Systems biology is increasingly recognized for its importance to infectious disease research. From a systems-level perspective, microbe-host interactions can be better understood by taking into account the dynamical molecular networks that constitute a biological system. Reconstructed networks are modeled and refined, undergoing iterative cycles of perturbation and experimental validation. Similar to the progressive nature of the analyses, the field itself is constantly evolving. This issue of Future Microbiology highlights new developments in the field, including emerging technologies impacting 'omic' analyses, new applications for modeling schemes, and discoverybased endeavors seeking to uncover novel pathogens and virulence factors. Regrettably, 'systems biology' is often used as a blanket term, mistakenly referenced in studies utilizing only highthroughput technologies or incorporated into titles to give extra weight or novelty [1]. Integration of multiple high-throughput data types represents just a single dimension of the field necessary to elucidate host responses to infection.

What truly sets systems biology apart from these correlation studies is the use of bioinformatics to computationally model molecular networks and define relationships between pathogen and host. There are several definitions of systems biology. Some emphasize that modeling and simulation be tightly integrated with experimentation to characterize network behavior [2], while others believe that systematic perturbation of individual network components will better define relationships governing pathway function [3]. The unifying theme to these different view points is the use of computational strategies modeling information obtained from experimental models, data types and stimuli (e.g., infectious agents). With respect to infectious diseases, bioinformaticists model molecular networks to make predictions about network behavior to a pathogen. Mathematical modeling, together with documented virus-host interaction data, can be used to predict key network components and/or connections (e.g., 'bottlenecks'), which can then be assessed by introducing targeted perturbations and monitoring the effects of these changes on the network as a whole. Subsequent analysis of model-based predictions, using siRNA knock-down studies or knock-out animal models, relate model findings to infection phenotypes and disease outcome in these experimental systems, refining models and driving further predictions (FIGURe 1).

To understand the true complexity of pathogen-host interactions, it is necessary for systems biology to extend into more complex experimental models and incorporate a greater breadth of high-throughput data. Small-scale studies have excelled our understanding of innate immune defenses, including recent characterization of the inflammasome as an intracellular pathogen sensor capable of activating caspase-1 [4], and $\mathrm{P} 8^{\mathrm{IPK}}$ as a cellular inhibitor of host defenses during influenza infection [5]. However, these model systems do not portray a comprehensive view of infection. Host antimicrobial responses, and conceivably the underlying mechanism(s) by which a pathogen blocks and/or modulates the activity of these responses, will be revealed in more complex models (e.g., nonhuman primates), aiding translational research efforts in rational drug design and vaccine development. In the case of influenza virus, macaques infected with pathogenic influenza viruses, including the fully reconstructed 1918 influenza virus [6] and, more recently, with the highly pathogenic avian influenza $\mathrm{H} 5 \mathrm{~N} 1$ virus [7], indicate that the host response occurs early and results in enhanced levels of innate immune signaling that remain unabated. Information obtained from these studies provide a foundation to build upon, using systems biology approaches, with the hope of defining host mediators of early dysregulated host responses dictating later stages of immunopathology. Recent work from our laboratory, using systems biology to model the impact of HCV infection on global metabolism, revealed

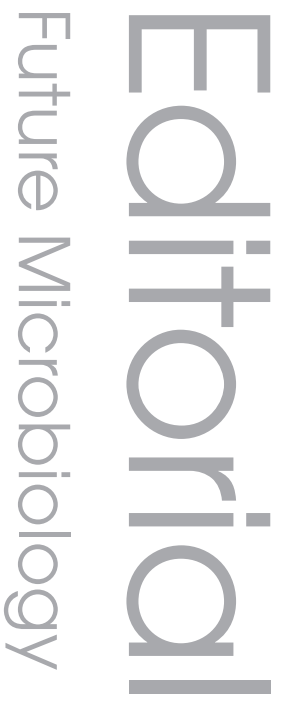

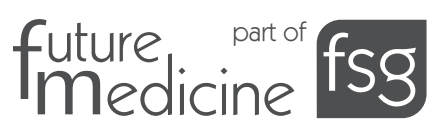

${ }^{\mathbf{6}}$ To understand the

true complexity of pathogen-host

interactions, it is necessary

for systems biology to extend into more

complex experimental

models and incorporate

a greater breadth of

high-throughput data." 


"Mathematical modeling
of interaction networks is
essential to allow
researchers to better
relate changes at the
molecular level to the
global properties
observed within a
biological system
during infection."

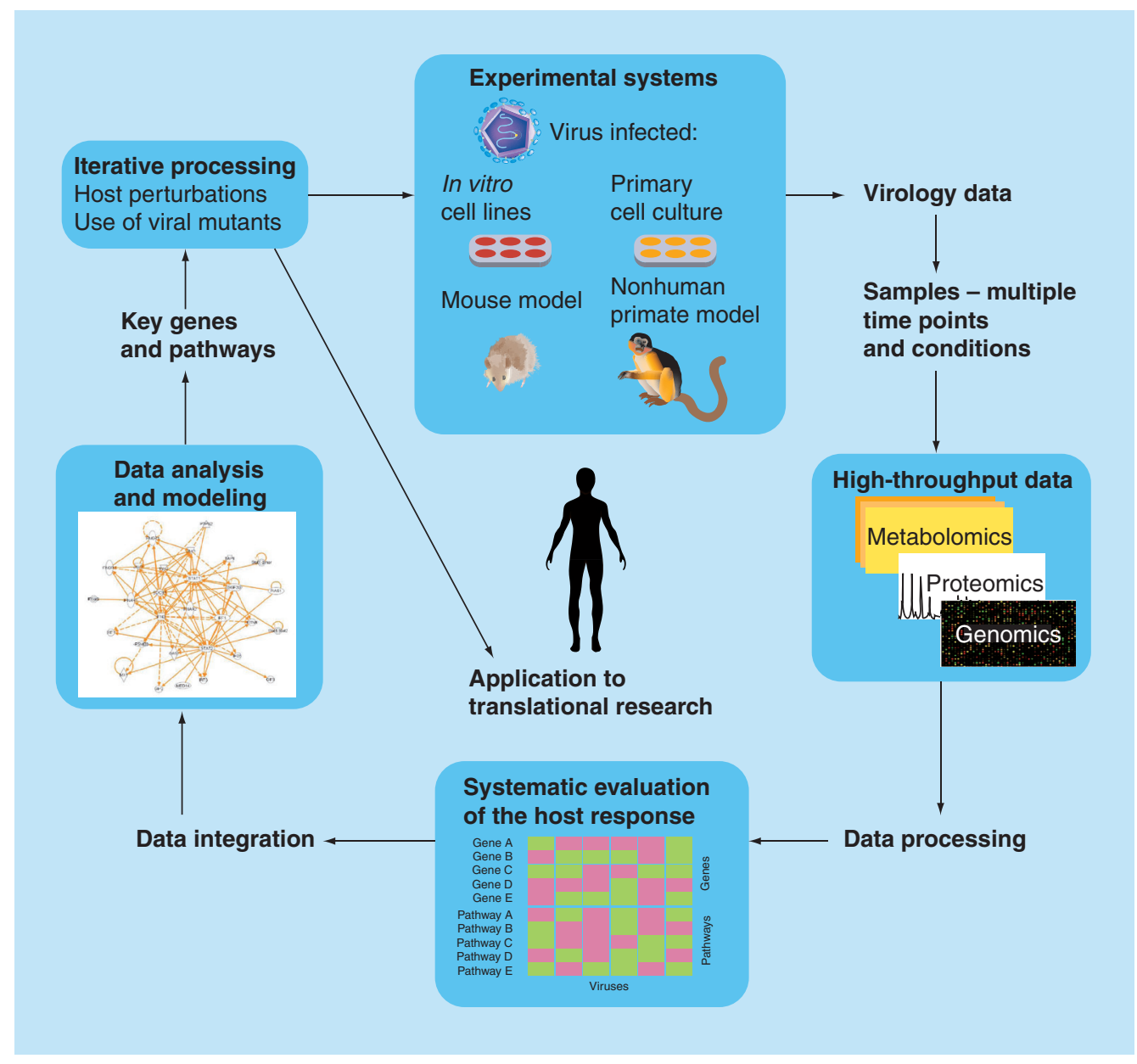

Figure 1. Iterative cycles of perturbation biology. Identification of key genes and pathways through computational modeling schemes of diverse data types.

that HCV exploits the metabolic resources of liver cells to support viral replication, a process that results in massive disruption of liver cell metabolism [8]. Mathematical modeling strategies were used to analyze $\mathrm{HCV}$-induced changes in the protein and lipid composition of liver cells, identifying proteins that are central points for connecting and controlling metabolic pathways. This approach led to the identification of mitochondrial fatty acid enzymes as key cellular proteins targeted by HCV. The same metabolic changes observed in liver cells were also found in liver biopsy specimens taken from HCV-infected liver transplant patients that had rapid recurrence of liver fibrosis, suggesting that similar disturbances in metabolic homeostasis occur during natural infection and may contribute to liver disease progression.

High-throughput analytical techniques are constantly evolving, allowing for greater coverage in cataloguing molecular components at RNA and protein levels. Emerging technologies, such as RNA-Seq, will greatly enrich current data sets by enhancing the detection of noncoding RNAs, including miRNAs, which are receiving a great deal of attention in the virology community, given their potential regulatory role during infection [9-11]. More recently, RNA-Seq has been applied to microbial systems, unveiling the transcriptome of Chlamydia trachomatis [12] and further defining the two-component regulatory system of Salmonella enterica serovar Typhi, involving OmpR [13]. However, a major pitfall of RNA-Seq is that over $70 \%$ of the reads map to rRNA making it particularly challenging to analyze the bacterial transcriptome. Recently, Armour and coworkers published a deepsequencing technique termed 'not-so-random' priming that significantly reduces the number of reads mapping to rRNA, potentially making it easier to distinguish microbial RNA species from host cell transcripts [14]. Microarrays are diversifying - no longer are they predominantly used for transcriptional profiling. Platforms are being developed to probe protein and glycolipid repertoires of infectious agents [15,16], as described by 
Felgner and coworkers and Twine and coworkers, respectively, in this issue of Future Microbiology. We have demonstrated that use of microarrays to profile host miRNA expression patterns, when combined with modeling, allows identification of HCV-associated miRNA-mRNA regulatory modules that are revealed through inverse expression relationships between miRNA and mRNAs and computational target predictions [17].

It is important to take a global perspective when investigating infection, and a systems biology approach to infectious disease research will permit greater understanding of the interplay between host and pathogen. Mathematical modeling of interaction networks is essential to allow researchers to better relate changes at the molecular level to the global properties observed within a biological system during infection. As new technologies develop and become readily available, the repertoire of information that can enrich modeling efforts will expand. This will likely translate into more effective strategies devised to counter pathogen-mediated cellular alterations, which can consequently be the primary cause of virus pathogenesis, for example, or set the stage for cellular processes to contribute to the pathological outcome. Unless systemslevel analysis is correctly pursued, the insights we hope to learn through this approach may be lost.

\section{Acknowledgement}

The authors thank Kaitlyn Grady for figure preparation and Alvin Go for enthusiastic assistance in manuscript editing.

\section{Financial \& competing interests disclosure}

Research in the authors' laboratory is supported by federal grants from the National Institute of Allergy and Infectious Diseases, NIH, Department of Health and Human Services under contract number HHSN272200800060C, and Public Health Service grants R01AI022646, R01HL080621, R24RR016354, P30DA015625, P01AI058113, and P51RR000166 from the National Institutes of Health, USA. The authors have no other relevant affiliations or financial involvement with any organization or entity with a financial interest in or financial conflict with the subject matter or materials discussed in the manuscript apart from those disclosed.

No writing assistance was utilized in the production of this manuscript.

\section{Bibliography}

1. Querec TD, Akondy RS, Lee EK et al.: Systems biology approach predicts immunogenicity of the yellow fever vaccine in humans. Nat. Immunol. 10(1), 116-125 (2009).

2. Cassman M: Barriers to progress in systems biology. Nature 438(7071), 1079 (2005).

3. Ideker $\mathrm{T}$, Thorsson $\mathrm{V}$, Ranish JA et al: : Integrated genomic and proteomic analyses of a systematically perturbed metabolic network. Science 292(5518), 929-934 (2001).

4. Muruve DA, Pétrilli V, Zaiss AK et al:: The inflammasome recognizes cytosolic microbial and host DNA and triggers an innate immune response. Nature 452(7183), 103-107 (2008).

5. Goodman AG, Smith JA, Balachandran O et al: : The cellular protein P58IPK regulates influenza virus mRNA translation and replication through a PKR-mediated mechanism. J. Virol. 81(5) 2221-2230 (2007).

6. Kobasa D, Jones SM, Shinya K et al.: Aberrant innate immune response in lethal infection of macaques with the 1918 influenza virus. Nature 445(7125), 319-323 (2007).

7. Baskin CR, Bielefeldt-Ohmann H, Tumpey TM et al:: Early and sustained innate immune response defines pathology and death in nonhuman primates infected by highly pathogenic influenza virus. Proc. Natl Acad. Sci. USA 106(9), 3455-3460 (2009).
8. Diamond DL, Syder AJ, Jacobs JM et al: Temporal proteome and lipidome profiles reveal hepatitis $C$ virus-associated reprogramming of hepatocellular metabolism and bioenergetics. PLoS Pathog. 6(1), e1000719 (2010).

9. Lu LF, Liston A: MicroRNA in the immune system, microRNA as an immune system. Immunology 127(3), 291-298 (2009).

10. Wang Z, Gerstein M, Snyder M: RNA-Seq: a revolutionary tool for transcriptomics. Nat. Rev. Genet. 10(1), 57-63 (2009).

11. Li Y, Chan EY, Li J et al.: MicroRNA expression and virulence in pandemic influenza virus infected mice. J. Virol. (2010) (In press).

12. Albrecht $\mathrm{M}$, Sharma CM, Reinhardt R et al.: Deep sequencing-based discovery of the Chlamydia trachomatis transcriptome. Nucleic Acids Res. (2009) (Epub ahead of print).

13. Perkins TT, Kingsley RA, Fookes MC et al.: A strand-specific RNA-Seq analysis of the transcriptome of the typhoid bacillus Salmonella typhi. PLoS Genet. 5(7), e1000569 (2009).

14. Armour CD, Castle JC, Chen R et al.: Digital transcriptome profiling using selective hexamer priming for cDNA synthesis. $N a t$. Methods 6(9) 647-649 (2009).

15. Vigil A, Davies DH, Felgner PL: Defining the humoral immune response to infectious agents using high-density protein microarrays. Future Microbiol. 5(2), 241-251 (2010).
16. Reid CW, Fulton KM, Twine SM: Never take candy from a stranger: the role of the bacterial glycome in host-pathogen interactions. Future Microbiol. 5(2), 267-288 (2010).

17. Peng X, Li Y, Walters KA et al.: Computational identification of hepatitis $\mathrm{C}$ virus associated microRNA-mRNA regulatory modules in human livers. $B M C$ Genomics 10, 373 (2009).

\section{Affiliations}

- Jennifer R Tisoncik University of Washington, Department of Microbiology, Seattle, WA 98195-8070, USA

Tel.: +1 2067326120

Fax: +1 2067326056

tisoncik@u.washington.edu

- Michael G Katze

University of Washington, Department of Microbiology and Washington National Primate Research Center, Box 358070 , Seattle, WA, 98195-8070, USA

Tel.: +1 2067326135

Fax: +1 2067326056

honey@u.washingon.edu 